\title{
Spectral principal component analysis of dynamic process data
}

\author{
N.F. Thornhilla,*, S.L. Shah ${ }^{\mathrm{b}}$, B. Huang ${ }^{\mathrm{b}}$, A. Vishnubhotla ${ }^{\mathrm{c}}$ \\ ${ }^{a}$ Department of Electronic and Electrical Engineering, University College London, Torrington Place, London WC1E 7JE, UK \\ ${ }^{\mathrm{b}}$ Department of Chemical and Materials Engineering, University of Alberta, Edmonton, Alberta, Canada T6G $2 G 6$ \\ ${ }^{\mathrm{c}}$ Matrikon Consulting Inc, Edmonton, Alberta, Canada T5N $4 \mathrm{A3}$
}

Received 21 June 2000; accepted 11 January 2002

\begin{abstract}
This article describes principal component analysis (PCA) of the power spectra of data from chemical processes. Spectral PCA can be applied to the measurements from a whole unit or plant because spectra are invariant to the phase lags caused by time delays and process dynamics. The same comment applies to PCA using autocovariance functions, which was also studied. Two case studies are presented. One was derived from simulation of a pulp process. The second was from a refinery involving 37 tags. In both cases, PCA clusters were observed which were characterised by distinct spectral features. Spectral PCA was compared with PCA using autocovariance functions. The performance was similar, and both offered an improvement over PCA using the time domain signals even when time shifting was used to align the phases. (C) 2002 Elsevier Science Ltd. All rights reserved.
\end{abstract}

Keywords: Autocorrelation; Autocovariance; Chemical industry; Dynamic system; Multivariate analysis; Principal component; Process operation; Power spectrum; Spectral analysis; Time delay

\section{Introduction}

It is important to detect and to diagnose the causes of oscillations in process operation because a plant running close to a product quality limit is more profitable than a plant that has to back away because of variations in the product (Martin, Turpin, \& Cline, 1991). This paper addresses the detection of oscillations and other types of time-varying behaviour when the measurements have time delays or phase lags. It presents and demonstrates methods for multivariate analysis using transforms of the data, such as the power spectrum and autocovariance functions, which are invariant to time delays and phase lags.

A previous approach to alignment of data trends used dynamic time warping (Gollmer \& Posten, 1996; Kassidas, Taylor, \& MacGregor, 1998). Kassidas et al. used that method with principal component analysis (PCA) for recognition of faults having different time duration. Lags and delays in measurements caused by process dynamics have also been addressed in PCA by the method of time shifting. The columns of the data

*Corresponding author. Tel.: + 44-20-7679-3983; fax: +44-20-73889325.

E-mail address: n.thornhill@ee.ucl.ac.uk (N.F. Thornhill). matrix representing tags with time delays are either time shifted, or the matrix is augmented with one or more time shifted replicates of the vector (see Wise and Gallagher, 1996, for a summary of the method). Time shifting aids PCA modelling for disturbance detection (Wachs \& Lewin, 1999), and additionally can identify dynamic relationships in the form of a time series model (Wise \& Ricker, 1992; Ku, Storer, \& Georgakis, 1995), or a dynamic model using partial least squares (PLS) modelling of input-output data (Lakshminarayanan, Shah, \& Nandakumar, 1997; Chen, McAvoy, \& Piovoso, 1998). Negiz and Cinar $(1997,1998)$ exploited past process measurements in PCA in a canonical variate state space model. Shi and MacGregor (2000) reviewed techniques for multivariate dynamic process modelling and gave a comparison of latent variable and subspace methods for reduction of the large lagged variables matrix.

Near infrared spectra (NIR) and infrared spectra are routinely analysed by PCA or used in PLS models for estimation of analyte concentrations in unknown samples (Karstang \& Henriksen, 1992; Riley, Rhiel, Zhou, Arnold, \& Murhammer, 1997; Yeung, Hoare, Thornhill, Williams, \& Vaghjiani, 1999), while Seasholtz (1999) described the impact of multivariate analysis of NIR and other spectroscopy at Dow Chemical. In those 
cases the instruments themselves created the spectra, but the spectra may also be calculated numerically. For instance, multivariate analysis of the spectra calculated from acoustic signals was reported by Belchamber and Collins (1993) who gave examples of the classification of the acoustic spectra in 24 frequency channels from a pump and an industrial blender. Tabe, Chow, Tan, Zhang, and Thornhill (1998) presented an application of acoustic spectral PCA to monitoring of fermentation process equipment. The contribution of this paper is to formulate the techniques of spectral PCA for use with process data and to present examples of their application.

Reported uses of PCA with transforms of the data matrix have focussed on multiscale wavelet transforms for on-line multivariate statistical process monitoring. Bakshi and Stephanopoulos (1994) and Bakshi (1998) used wavelets and other templates for multiscale representation of process data in combination with PCA for modelling of data having features that changed over time and frequency. Kosanovich and Piovoso (1997), Luo, Misra, and Himmelblau (1999) and Shao, Jia, Martin and Morris (1999) have also had success with multiscale PCA using wavelets. Stork, Veltkamp, and Kowalski (1998) applied wavelet transforms to NIR spectra in order to detect spectral features characterised by wavelength and scale. Wavelet functions are localised, however, so the wavelet transform is not invariant to time delays.

Oscillatory disturbances are not localised in time and thus the Fourier transform and power spectrum provide a natural means for their analysis. Harris, Seppala, Jofreit, and Surgenor (1996) reported plant-wide control loop assessment in which they found the spectral analysis of the univariate trends to be useful. Hägglund (1995) described a method for the on-line detection of oscillations within a control loop and other authors have also considered this problem (Rengaswamy \& Venkatasubramanian, 1995; Thornhill \& Hägglund, 1997). Other applications utilising spectra have included paper making (Bissessur, Martin, \& Morris, 1999).

Multivariate spectral analysis has several advantages over analysis in the time domain. It gives improved signal-to-noise ratio if the spectral content of the wanted signal occupies a narrow band of frequencies compared to the noise. The power spectrum is invariant to time delays or phase shifts caused by process dynamics. It is also insensitive to missing values and outliers because the transforms of such effects are spread thinly across all frequencies in the spectrum.

This analysis was used for retrospective study of an ensemble of data collected over a period of time in order to highlight clusters of measurements that were affected by the same oscillation during that period. It was found that the method was also able to detect dynamic disturbances having a non-oscillatory nature. Identification of oscillatory and other clusters together with process insight and a knowledge of the plant layout gave an understanding of the propagation of disturbances through the plant.

Section 2 presents the methods for spectral PCA and for PCA using autocovariance functions and introduces two case studies. One was a challenge problem provided courtesy of EnTech Control Inc., Toronto, Ont., Canada. The other was a larger scale study courtesy of a SE Asian refinery where the aim was to detect and characterise the disturbances. Section 3 discusses results from the case studies and highlights some benefits of the new approaches. A comparison with time-shift PCA is presented in section 4, where it is also shown how the optimum shifts were determined from the phases of the spectra. The paper ends with concluding remarks.

\section{Methods}

\subsection{PCA methods and data treatments}

\subsubsection{Spectral PCA-formulation}

Descriptions of PCA may be found from many sources, for example in Chatfield and Collins (1980), Wold, Esbensen, \& Geladi (1987). Applications to process data have also been reported, for instance by Wise, Ricker, Veltkamp, and Kowalski (1990), Kresta, MacGregor, and Marlin (1991), Wise and Gallagher (1996) and Goulding, Lennox, Sandoz, Smith, and Marjanovic (2000). The novel feature of spectral PCA is that the rows of the data matrix, $\boldsymbol{X}$, are the single-sided power spectra $P(f)$ of the signals over a range of frequencies up to the Nyquist frequency (one-half of the sampling frequency). If the data sets are so large that computing speed or memory is an issue then smoothed single-sided power spectra may be calculated using the averaged periodogram method (Welch, 1967). In that case the number of frequency channels, $N$, may be considerably fewer than the original number of data points and therefore data compression is achieved. If compression is not required then the fast Fourier transform may be used, for instance using MATLAB (The Mathworks, Natick, MA):

$N$ frequency channels $\rightarrow$

$\boldsymbol{X}=\left(\begin{array}{ccc}P_{1}\left(f_{1}\right) & \cdots & P_{1}\left(f_{N}\right) \\ \cdots & \cdots & \cdots \\ P_{m}\left(f_{1}\right) & \cdots & P_{m}\left(f_{N}\right)\end{array}\right) \begin{gathered}m \text { process } \\ \text { variables } \\ \downarrow\end{gathered}$.

A full PCA decomposition reconstructs the $X$ matrix as a sum over $m$ orthonormal basis functions $\boldsymbol{w}_{1}{ }_{1}$ to $\boldsymbol{w}_{m}^{\prime}$ which are spectrum-like functions having $N$ frequency channels arranged as a row vector: 
$\boldsymbol{X}=\left(\begin{array}{c}t_{1,1} \\ \cdots \\ t_{m, 1}\end{array}\right) \boldsymbol{w}_{1}^{\prime}+\left(\begin{array}{c}t_{1,2} \\ \cdots \\ t_{m, 2}\end{array}\right) \boldsymbol{w}_{2}^{\prime}+\cdots+\left(\begin{array}{c}t_{1, m} \\ \cdots \\ t_{m, m}\end{array}\right) \boldsymbol{w}_{m}^{\prime}$

The $\boldsymbol{w}_{i}^{\prime}$-vectors are the normalised eigenvectors of the $N \times N$ matrix $\boldsymbol{X}^{\prime} \boldsymbol{X}$. They are ordered according to the size of the eigenvalues of $\boldsymbol{X}^{\prime} \boldsymbol{X}$. The ratio between the eigenvalue and the sum of all the eigenvalues gives a measure of the total spectral variation captured by that eigenvector.

The above expression may be written compactly as $\boldsymbol{X}=\boldsymbol{T} \boldsymbol{W}^{\prime}$ where the $i$ th column of $\boldsymbol{T}$ is $\left(\begin{array}{lll}t_{1, i} & \cdots & t_{m, i}\end{array}\right)^{\prime}$ and the rows of $\boldsymbol{W}^{\prime}$ are $\boldsymbol{w}_{1}^{\prime}$ to $\boldsymbol{w}_{m}^{\prime}$. The orthonormality of the rows of $\boldsymbol{W}^{\prime}$ means that $\boldsymbol{T}=\boldsymbol{X} \boldsymbol{W}$. Singular value decomposition $\boldsymbol{X}=\boldsymbol{U} \boldsymbol{D} \boldsymbol{V}^{\prime}$ provides a means for computation of the requisite vectors with $\boldsymbol{T}=\boldsymbol{U} \boldsymbol{D}$ and $\boldsymbol{W}^{\prime}=$ $\boldsymbol{V}^{\prime}$. Matrix $\boldsymbol{D}$ is diagonal and its elements are the positive square roots of the eigenvalues of $\boldsymbol{X}^{\prime} \boldsymbol{X}$.

A description of the majority of the variation in $\boldsymbol{X}$ can often be achieved by truncating the PCA description. If all the variables had similar spectral features then one term (i.e. one principal component) would describe most or all of the variability in the spectra. In other cases more terms may be needed. The following is a three-PC model in which the variation of $\boldsymbol{X}$ that is not captured by the first three principal components appears in an error matrix $\boldsymbol{E}$ :

$\boldsymbol{X}=\left(\begin{array}{c}t_{1,1} \\ \cdots \\ t_{m, 1}\end{array}\right) \boldsymbol{w}_{1}^{\prime}+\left(\begin{array}{c}t_{1,2} \\ \cdots \\ t_{m, 2}\end{array}\right) \boldsymbol{w}_{2}^{\prime}+\left(\begin{array}{c}t_{1,3} \\ \cdots \\ t_{m, 3}\end{array}\right) \boldsymbol{w}_{3}^{\prime}+\boldsymbol{E}$.

The issue of the correct number of terms is discussed by Chatfield and Collins (1980), Valle, Li, and Qin (1999) and elsewhere. The case studies needed one, two or three terms, the decision to truncate being made when the eigenvalue associated with the next principal component represented $<5 \%$ of the sum of all the eigenvalues.

Each spectrum in $\boldsymbol{X}$ may be represented graphically. For instance, when three $\boldsymbol{w}^{\prime}$-vectors are in use the $i$ th spectrum maps to a point having the coordinates $t_{i, 1}, t_{i, 2}$ and $t_{i, 3}$ in a three-dimensional space called a scores plot. Similar spectra have similar $t$-coordinates. Therefore such groups form clusters. When two $\boldsymbol{w}^{\prime}$-vectors are used, the scores plot is two dimensional.

The rows of the error matrix, $\boldsymbol{E}$, are the unmodelled part of the spectra in $\boldsymbol{X}$. Therefore the magnitudes of the rows of $\boldsymbol{E}$ should be inspected. The following vector of squared prediction errors (SPE) can be used in which each term is the sum of squares of the elements of a row of $\boldsymbol{E}$ (Jackson \& Mudholkar, 1979; Wise et al., 1990; Kourti \& MacGregor, 1996):
$\boldsymbol{S P E}=\left(\begin{array}{c}S P E_{1} \\ S P E_{2} \\ \cdots \\ S P E_{m}\end{array}\right)=\left(\begin{array}{c}\sum_{i=1}^{N}\left(E_{1, i}\right)^{2} \\ \sum_{i=1}^{N}\left(E_{2, i}\right)^{2} \\ \cdots \\ \sum_{i=1}^{N}\left(E_{m, 1}\right)^{2}\end{array}\right)$.

A common reason why one particular spectrum may have a large $S P E_{i}$ value (i.e. it is not well modelled by a reduced number of principal components) is that it has spectral features at frequencies where no other spectrum shows any features.

When a reference data set from normal operation is available, such as in the case when PCA is used for online monitoring, then a statistical threshold value would be selected for the $S P E_{i}$ values based on the properties of the reference distribution. During on-line monitoring any tag that violates the threshold would be flagged as faulty. The purpose of the analysis in this paper, by contrast, is the examination of historical data sets containing both normal and abnormal process tags. Therefore, the only distribution of $S P E_{i}$ available was the empirical one for the whole data set. The distribution was plotted as a histogram of $S P E_{i}$ values and abnormal tags were taken to be those that were judged to be outliers in the histogram, as discussed in Sections 3.1 and 3.2.

\subsubsection{Autocovariance PCA-formulation}

An estimator for the autocovariance function of a zero-mean signal $y[i]$ having $n$ samples is shown below. The index $\ell$ is known as the lag:

$A(\ell)=\frac{1}{n-\ell} \sum_{i=\ell+1}^{n} y[i] y[i-\ell]$.

The autocovariance is the inverse Fourier transform of the two-sided power spectrum (the Wiener-Khinchin theorem, (Press, Flannery, Teukolsky, \& Vetterling, 1986)). Therefore, the advantages of the use of spectra highlighted in the introduction apply also to autocovariance functions. In particular, like power spectra, autocovariance functions are invariant to time lags and delays. For example, $\sin (\omega t)$ and $\cos (\omega t)$ have the same autocovariance function. Here the autocovariances were determined from the power spectra, and lags up to $N / 2$ were used where $N$ is the number of samples in the original data set. Only lags up to $N / 2$ are valid because the power spectrum is unique only up to one-half of the sampling frequency. Thus, the performance of PCA using autocovariance functions can be directly compared with spectral PCA because the singlesided power spectrum also has $N / 2$ frequency channels.

PCA using autocovariance functions was formulated in the same way as was described for spectral PCA except that the rows of the data matrix were the autocovariance functions for the time trends. In that case, the $\boldsymbol{w}^{\prime}$-vectors are an orthonormal set of basis 
functions for the autocovariance functions. PCA using the autocovariance functions is considered to be a spectral PCA method because the autocovariances are determined from the spectra.

\subsubsection{Data pre-processing and filtering of spectra}

It is usual in time domain PCA to mean centre the data sets and to scale to unit variance. In spectral PCA the following data pre-processing may be applied:

- Mean centre the time trend and remove linear trends before calculation of the spectra.

- Filter the spectra, if required.

- Scale the spectra to the same total power such that $\sum_{i=1}^{N} P\left(f_{i}\right)=$ constant

where $P\left(f_{i}\right)$ is the spectral power in the $i$ th channel. or

- Scale the autocovariance functions so that the covariance at zero lags is unity. For a zero mean signal the scaled autocovariance function is the same as the autocorrelation function.

The first of these steps removes steady offsets or linear ramps from the time domain data which would otherwise appear in the $f=0$ channel of the spectrum.

The spectrum may be filtered by replacing the values in selected frequency channels with zeros, i.e. an optimum Wiener filter for the case where the wanted signal and the interfering signal lie in separate regions of the spectrum (Press et al., 1986). The ease of such an approach is a benefit of spectral PCA in contrast to filtering in the time domain where design of a narrow-band filter is a skilled task. Selective removal of frequency components can highlight subtle effects, for instance, when a dominant oscillation masks the presence of a small oscillation at a different frequency. It can also remove non-stationary low-frequency trends such as those caused by daily temperature variations. For the autocovariance functions, the interference must be removed from both positive and negative frequencies in the two-sided power spectrum before the inverse FFT (Thornhill, Huang, \& Zhang, 2002).

The scaling steps are equivalent to scaling to constant variance in the time domain because the signal variance is proportional to total power. Alternatively the spectra may be left unscaled if a meaning can be attributed to the intensity of the signal.

\subsubsection{Practical computations}

Once the spectra have been constructed with the above pre-processing steps then spectral PCA can proceed using an industry standard package such as PLS_Toolbox 2.1 (Eigenvector Research, Manson, WA,
USA), ProcessMonitor (Matrikon, Edmonton, Canada), SIMCA-P (Umetrics AB, Umeå, Sweden), or Unscrambler (CAMO ASA, Oslo, Norway). The option to mean centre the $\boldsymbol{X}$ matrix should be disabled because in spectral PCA the mean centring is applied to the time trend data before Fourier transformation.

\subsubsection{Time-shift PCA}

The method of lagged variables has been used in time domain PCA to address the problems of time lags and delays. Wise and Gallagher (1996) gave an overview of the procedure. If the time delays are known then time trends in the rows of the $\boldsymbol{X}$ matrix may be replaced by time delayed trends:

$$
\boldsymbol{X}=\left(\begin{array}{ccc}
n \text { samples } & \rightarrow \\
x_{1}\left(t_{1}\right) & \cdots & x_{1}\left(t_{n}\right) \\
x_{2}\left(t_{1}-\tau_{2}\right) & \cdots & x_{2}\left(t_{n}-\tau_{2}\right) \\
\cdots & \cdots & \cdots \\
x_{m}\left(t_{1}-\tau_{m}\right) & \cdots & x_{m}\left(t_{n}-\tau_{m}\right)
\end{array}\right) \text { variables }, \downarrow
$$

where the row vectors $x_{i}$ are the time trends of $m$ measurements having $n$ samples and $\tau_{2}$ to $\tau_{m}$ are the time shifts of the second to $m$ th measurements. The time shifts are relative and can be negative. For instance, $\tau_{i}$ would be negative if a feature in the $i$ th time trend occurred before a similar feature in the time trend in the first row. In the case of a single plant-wide oscillation, alignment of the measurements using the correct time delays would enable a one-PC model to describe most or all of the variability. The $\boldsymbol{w}^{\prime}$-vector would be a periodic time domain function with the same frequency as the oscillating measurements.

The arguments of the Fourier transforms of the time trends can be used to determine the optimum time shifts for the case when the time trends are oscillating with a single frequency (see Section 4). If the angular frequency of oscillation is $\omega_{\text {osc }}$ then the phase difference between the $i$ th process variable and the first is:

$\alpha_{i}=\angle X_{i}\left(\mathrm{j} \omega_{o s c}\right)-\angle X_{1}\left(\mathrm{j} \omega_{o s c}\right)$,

where $\angle X_{i}\left(\mathrm{j} \omega_{\text {osc }}\right)$ is the argument of the complex Fourier transform of $x_{i}(t)$ at the frequency of the oscillation (i.e. at the frequency where the power spectrum has its peak). The frequency response of a pure delay $T_{d}$ is $\mathrm{e}^{-\mathrm{j} \omega T_{d}}$. This is a complex quantity having unity magnitude and argument $-\omega T_{d}$, meaning that the phase of the delayed signal relative to the undelayed signal is $-\omega T_{d}$. Therefore, the time delay of the $i$ th process variable relative to the first is:

$T_{d_{i}}=-\frac{\alpha_{i}}{\omega_{o s c}}$ 


\subsection{Case studies}

\subsubsection{Challenge problem}

Data trends from a simulation of a pulp process kindly provided by EnTech Control Inc. were posed as a challenge to detect simulated faults in the control system of the plant. Hardwood and softwood pulps are mixed to give a stream of a desired composition. There were 1934 samples from 12 process measurements (tags) each of which was associated with a control loop. Some features of the control system are outlined in Table 1 and the process schematic is shown in Fig. 1. The time trends, normalised spectra and autocovariance functions of the controller errors (set point-process variable) are shown in Fig. 2. The spectra were normalised such that the total power (the area under the curve) was the same in each. The reason why the magnitude of the normalised spectrum for tag 2 was small is that the spectrum contained noise spread across all frequencies, while in all other cases the signal power was concentrated in just a few frequency channels. The autocovariance functions were all unity at zero lags, but in the case of tag 2 the autocovariance was close to zero when the lag was one or more, as expected for a random signal.

It can be seen that there were two significant oscillations, one at $0.002 \mathrm{~min}^{-1}$ (an oscillation having a period of $500 \mathrm{~min}$ ) in tags $3-10$ and 12 , and one at $0.02 \mathrm{~min}^{-1}(50 \mathrm{~min}$ oscillation) in tags 1 and 11 . The origin of the $0.002 \mathrm{~min}^{-1}$ low-frequency oscillation is

Table 1

Features of the control system from the challenge problem

\begin{tabular}{cl}
\hline Tag & Special features \\
\hline 3 & Slave in cascade from tag 7 in ratio with tag 6 \\
6 & Slave in cascade from tag 7 in ratio with tag 3 \\
7 & Master in cascade onto the ratio controller tag $3 / \operatorname{tag} 6$ \\
8 & Influenced by tags 6 and 9 \\
9 & Slave in cascade from tag 10 \\
12 & Has feed forward from tag 11 \\
\hline
\end{tabular}
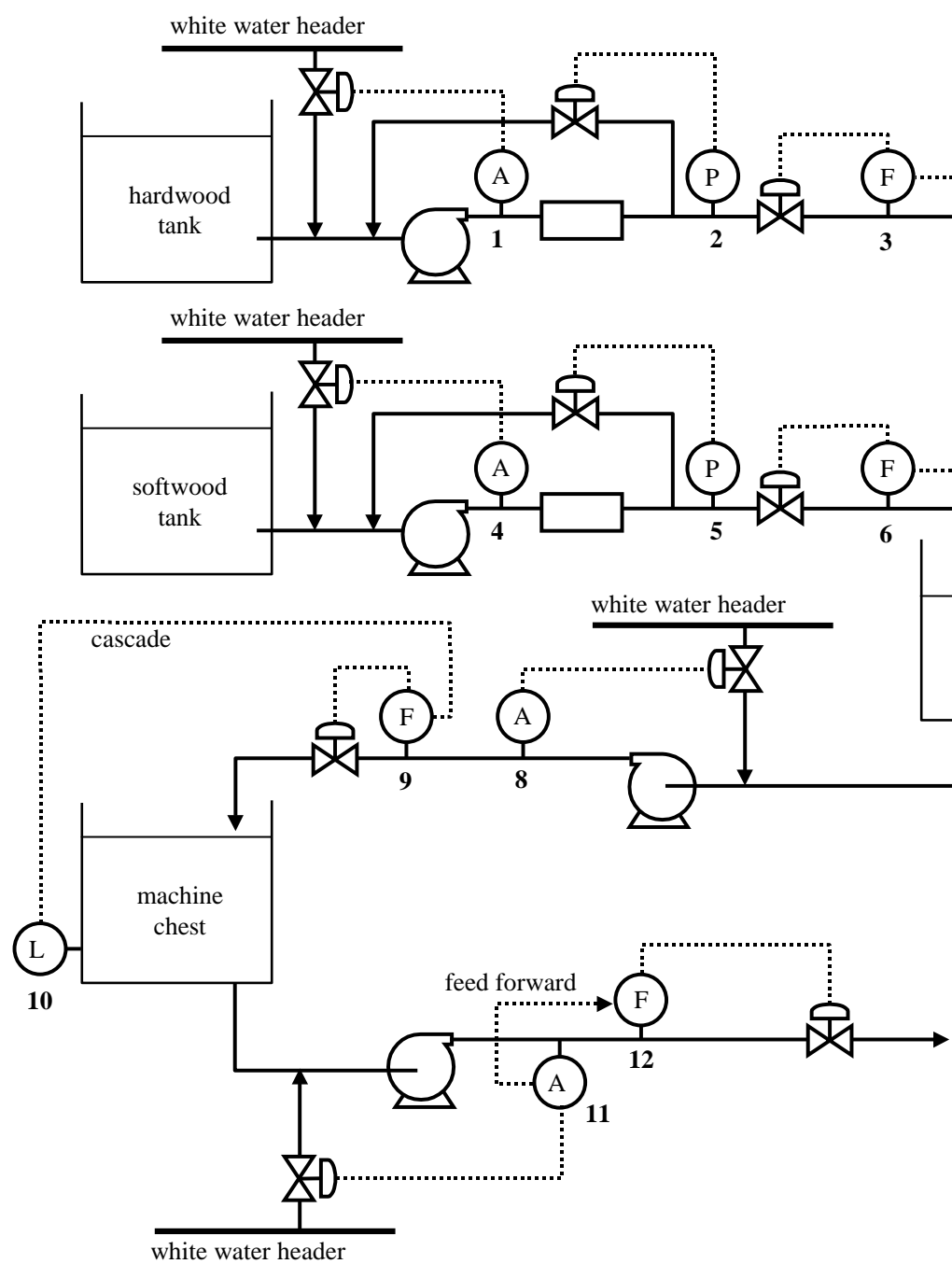

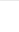



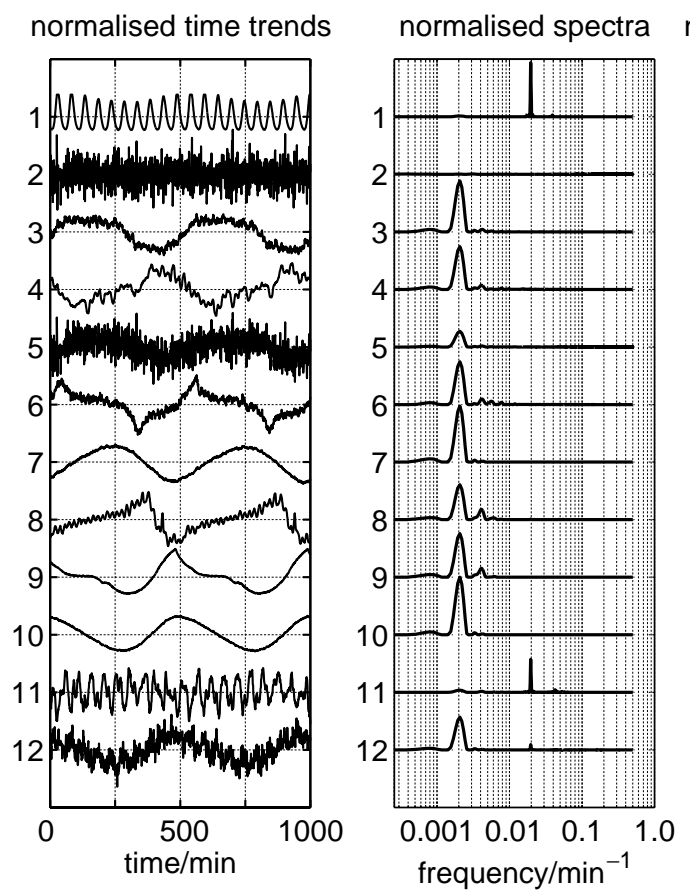

normalised autocovariances
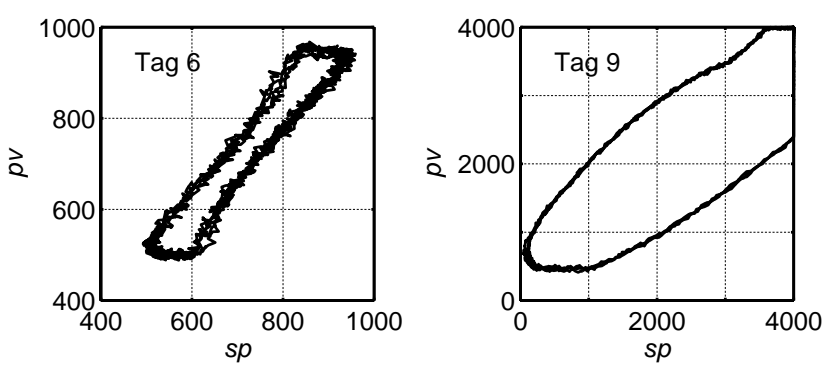

Fig. 2. Above: time trends, spectra and autocovariance functions for the challenge problem. Below: trajectories for the set-point versus process variable for tags 6 and 9 .

discussed in the next paragraph. The faster $0.02 \mathrm{~min}^{-1}$ oscillation originated in the composition control loop for tag 1 and it can also be seen in the time trends of the composition measurement at tag 11 . The time trend for tag 1 is that of a control loop oscillating at its cross-over frequency such as is seen during Ziegler Nichols tuning because the loop gain was set very high. The requirement of spectral PCA is that it should detect groups of tags characterised by these two oscillations and should answer queries such as whether trend 12 was influenced by both oscillatory disturbances.

The origin of the $0.002 \mathrm{~min}^{-1}$ oscillation was a sticking valve in the control loop for tag 6 . The plots of the spectra in Fig. 2 show that tag 6 had a series of smaller peaks to the right-hand side of the main spectral peak. These are harmonics and are generated in a control loop when non-linearities such as a valve faults or saturation lead to non-sinusoidal limit cycles (Cook, 1986; Åström, 1991; Thornhill \& Hägglund, 1997). The lower left panel in Fig. 2 shows a set point (sp) versus process variable (pv) map for loop 6 , which shows the pattern characteristic of a valve with hysteresis and a dead band. The oscillatory flow caused by loop 6 influenced the upstream pressure tag 5 and hence tag 4 . It affected the blend tank level, tag 7 , and also tag 3 because of the ratio controller. The composition controller, tag 8 , failed to regulate the oscillations in composition caused by the oscillatory flow from tag 6 . This varying composition in turn disturbed the flow through the valve in the control loop for tag 9 to the extent that it caused the set point to saturate, thus introducing additional non-linearity (lower right panel in Fig. 2). Based on this discussion, spectral PCA should highlight loops 6 and 9 because they have non-linearities and loop 8 because it is failing to regulate the consistency.

\subsubsection{Refinery example}

A larger example was provided courtesy of a SE Asian refinery. Measurements from 37 process tags from a hydrogen plant (Terrible et al., 1999) were sampled at $1 \mathrm{~min}$ intervals from the plant for $24 \mathrm{~h}$ of running. Fig. 3 
shows a simplified process schematic and Fig. 4 shows a 500 min snapshot of the time trends and the spectra of the controller errors. Some clusters can be observed by eye, for instance, the trends for tags 2, 3, 4, 10, 20, and
34 were all characterised by the same oscillation, while tags 35 and 36 also look similar to one another. The challenge for spectral PCA is to highlight those clusters and find any other members of the clusters that were not

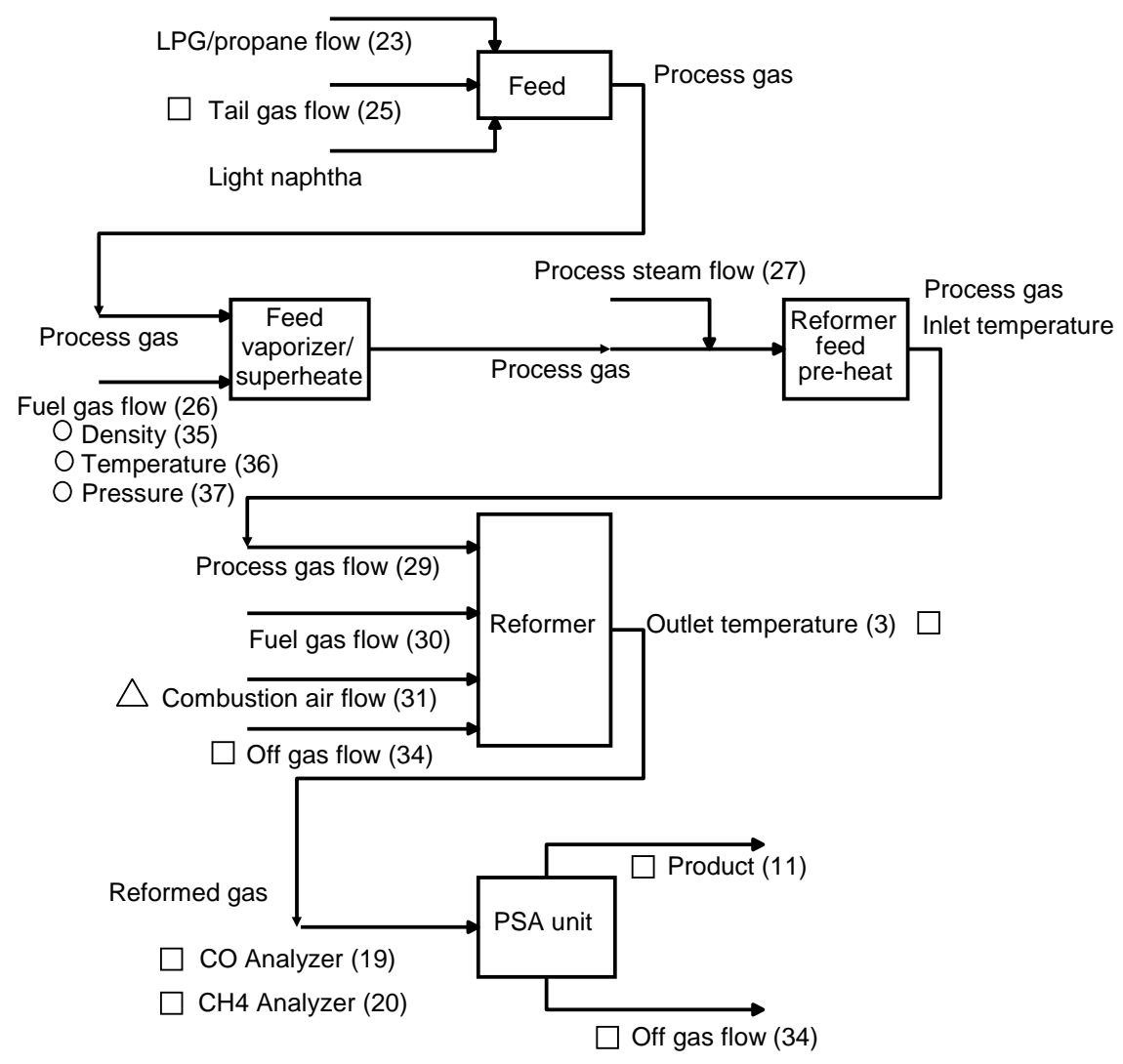

Fig. 3. Process schematic for the refinery example. The symbols indicate clusters of tags identified using spectral PCA.
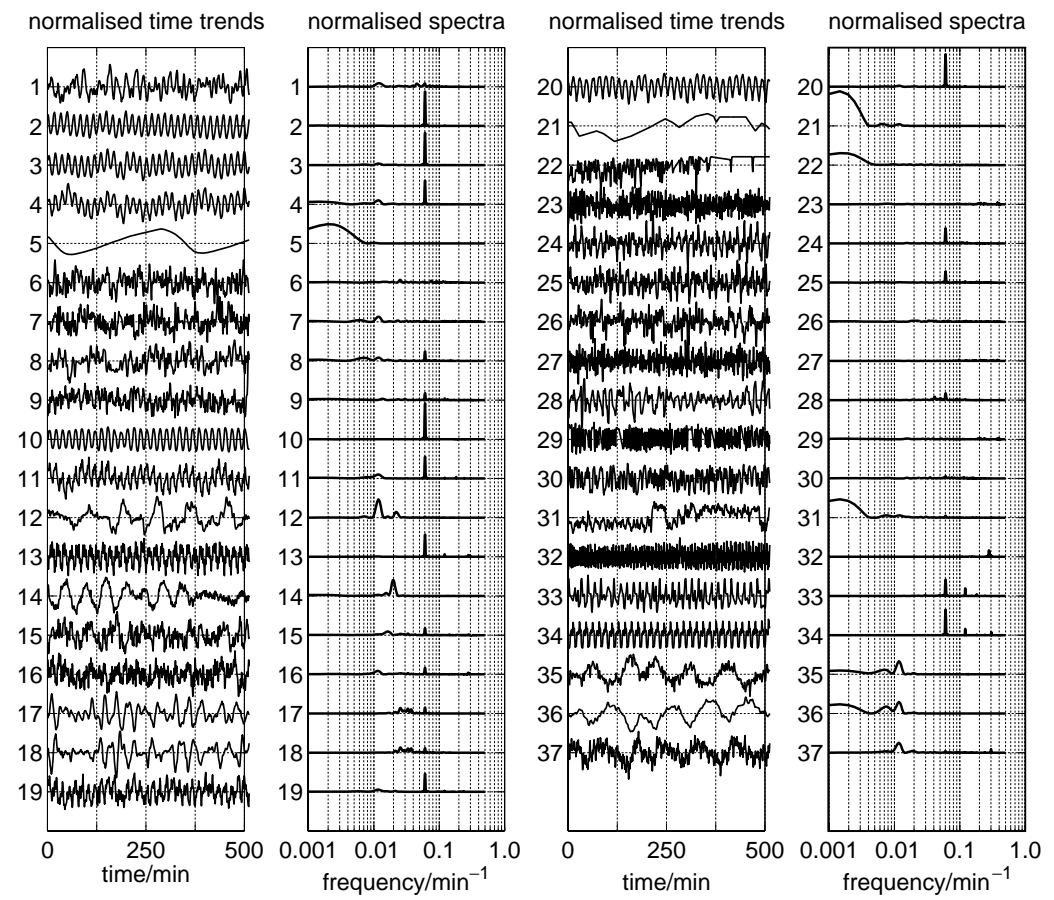

Fig. 4. Time trends and spectra for the refinery example. 
so easily detected by eye. This example illustrates an application of spectral PCA under industrial conditions.

\section{Results and discussion}

\subsection{Results from the challenge problem}

\subsubsection{Spectral PCA}

Table 2 shows the percentage of variance explained by spectral PCA in the pulp simulation challenge problem. The plots in column (a) of Fig. 5 show the results of PCA using the spectra from Fig. 2. The scores plot (top) shows the weightings, $t$, which when applied to the normalised $\boldsymbol{w}^{\prime}$-vectors shown in the middle plot approximately reconstructed the 12 spectra using a two-PC model in which $m=12$.

The $\boldsymbol{w}_{1}^{\prime}$-vector of the two-PC model had a spectral peak at a frequency of $0.002 \mathrm{~min}^{-1}$ (the $500 \mathrm{~min}$ oscillation). It also had a small secondary peak at $0.004 \mathrm{~min}^{-1}$ that represents the second harmonic of the oscillation. The $\boldsymbol{w}_{2}{ }_{2}$-vector had a spectral peak at $0.02 \mathrm{~min}^{-1}$ representing the oscillation with a period of $50 \mathrm{~min}$. Most of the tags were dominated by one or the other of the oscillations. Therefore, the points on the scores plot lie along the two orthogonal axes, those with the $0.002 \mathrm{~min}^{-1}$ oscillation on the horizontal axis (diamond symbols) while tags 1 and 11 with the $0.02 \mathrm{~min}^{-1}$ oscillation lie on or close to the vertical axis (circles). The requirement that spectral PCA should distinguish between the tags disturbed by the two oscillations has been achieved.

Tag 2 (square symbol) contained random noise and a flat frequency spectrum of small magnitude. It was not modelled by either $\boldsymbol{w}^{\prime}$-vector and thus appeared at the coordinates $(0,0)$ in the weightings plot. Tag 5 (triangle)

Table 2

Summary of PCA results

\begin{tabular}{llccc}
\hline & \multicolumn{4}{c}{ Percentage of variance explained by: } \\
\cline { 2 - 5 } & PC 1 & PC 2 & PC 3 & PC 4 \\
\hline Challenge problem & & & & \\
Spectral PCA & 80.4 & 18.7 & $(0.67)$ & \\
PCA of filtered spectrum & 58.3 & 37.9 & $(2.11)$ & \\
Autocovariance PCA & 80.4 & 18.7 & $(0.67)$ & \\
Refinery example & & & & \\
Spectral PCA & 66.7 & 16.6 & 7.08 & $(2.60)$ \\
Time domain PCA & 21.1 & 11.3 & 8.24 & 6.00 \\
Refinery example subset & & & & \\
Time domain PCA & 65.6 & 24.8 & 6.46 & $(2.03)$ \\
Time shifted PCA & 85.5 & 7.5 & $(4.39)$ & \\
Spectral PCA & 98.9 & $(0.76)$ & & \\
\hline
\end{tabular}

(a)
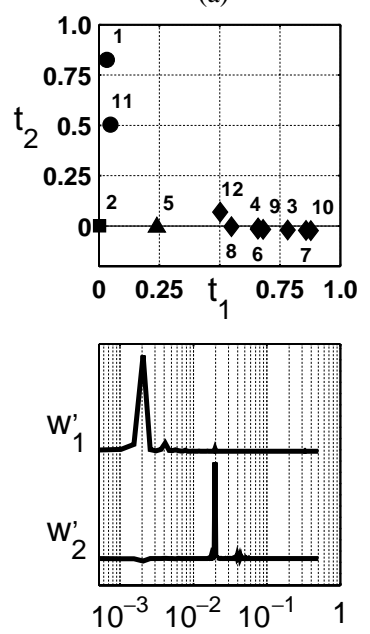

frequency/min $\mathrm{min}^{-1}$

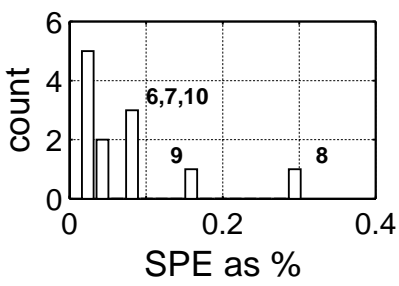

(b)
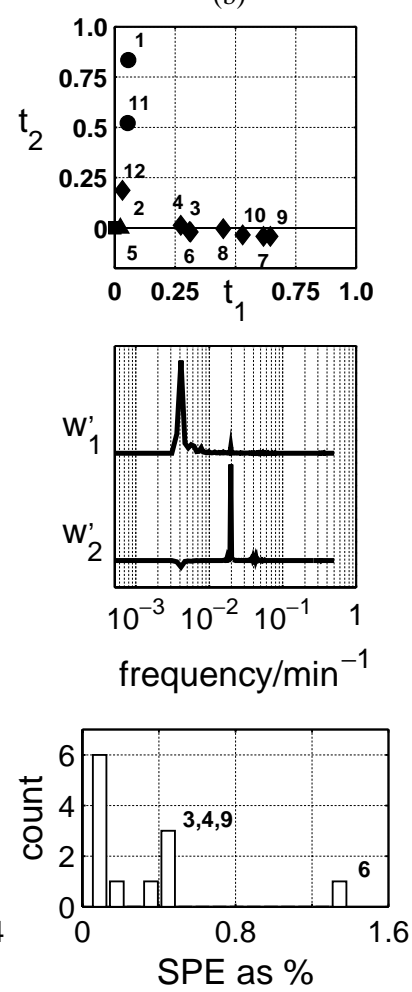

(c)
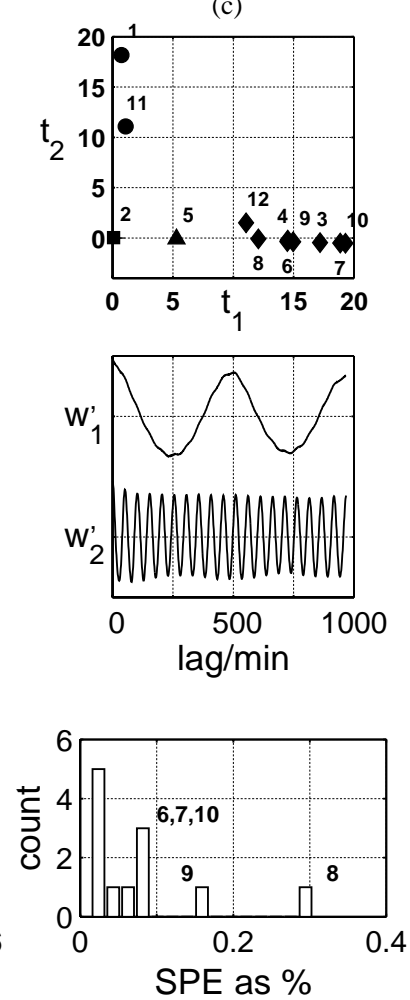

Fig. 5. Score plots (top), $\boldsymbol{w}^{\prime}$-vectors (middle) and SPE histograms (bottom) for the challenge problem: (a) spectral PCA analysis (b) spectral PCA with filtering (c) PCA using autocovariance functions. 
showed a small spectral peak at $0.002 \mathrm{~min}^{-1}$ and therefore lay on the horizontal axis but it was close to the origin because it also was noisy.

The lowest panel in column (a) of Fig. 5 shows a histogram of the distribution of the $S P E_{i}$ expressed as a percentage of the total variance in the data set. For instance, three tags $(6,7$ and 10$)$ all had $S P E$ values between $0.075 \%$ and $0.095 \%$ so the vertical bar at that position shows a tag count of three. Tag 8 was separated from the main distribution and thus was correctly classified as abnormal. The reason why tag 8 had a large $S P E$ value is because it had a larger second harmonic than could be captured by the $\boldsymbol{w}_{1}{ }_{1}$-vector. Tag 9 , which also was abnormal, was detected because it also had an $S P E$ value that was clearly separated from the main distribution. It was known from the sp-pv plot in Fig. 1 that tag 6 also had an abnormality. Tag 6 lies with tags 7 and 10 in a group that was only slightly separated in the $S P E$ histogram. Therefore its abnormality was not detected when using PCA on the full spectra and further investigation was needed.

\subsubsection{PCA using filtered spectra}

The PCA model using the full spectra from the pulp process was dominated by the spectral features at 0.002 and $0.02 \mathrm{~min}^{-1}$. The use of filtered spectra allows analysis at a higher resolution of the remaining features by selectively removing the dominating influence of a spectral feature. Thornhill et al. (2002) give an automated procedure for selection of the filter. The approach is similar in concept to disjoint modelling (Wold, 1976) because it inspects the variation that remains after major features have been accounted for. In column (a) of Fig. 5 tag 12 had a positive value for $t_{1}$ but also a non-zero value for $t_{2}$ which suggests that it had a small amount of the $0.02 \mathrm{~min}^{-1}$ oscillation superimposed on the $0.002 \mathrm{~min}^{-1}$ oscillation. A natural course of action, therefore, was to remove the low-frequency oscillation at $0.002 \mathrm{~min}^{-1}$ by spectral filtering, as described in the Methods section. Fig. 6 shows the filtered spectra and the filtered time trends. Because the dominant spectral peak at $0.002 \mathrm{~min}^{-1}$ was removed, the overall effect of filtering was to enhance the relative magnitude of the $0.02 \mathrm{~min}^{-1}$ oscillation and thus to increase the amount of variability explained by the $\boldsymbol{w}_{2}^{\prime}{ }_{2}^{-}$ vector (see Table 2).

The PCA results are shown in the plots in column (b) in Fig. 5. There, the $\boldsymbol{w}^{\prime}$-vectors were similar to those of the full spectral PCA analysis in column (a), except that the new $\boldsymbol{w}^{\prime}{ }_{1}$-vector had a peak at $0.004 \mathrm{~min}^{-1}$ rather than $0.002 \mathrm{~min}^{-1}$. Therefore it captured mainly the second harmonic of the $0.002 \mathrm{~min}^{-1}$ oscillation. The scores plot in column (b) shows that tag 12 had a larger value of $t_{2}$ when filtered spectra were used. The conclusion is that tag 12 was influenced by the higher frequency $0.02 \mathrm{~min}^{-1}$ oscillation. PCA using filtered normalised filtered trends normalised spectra
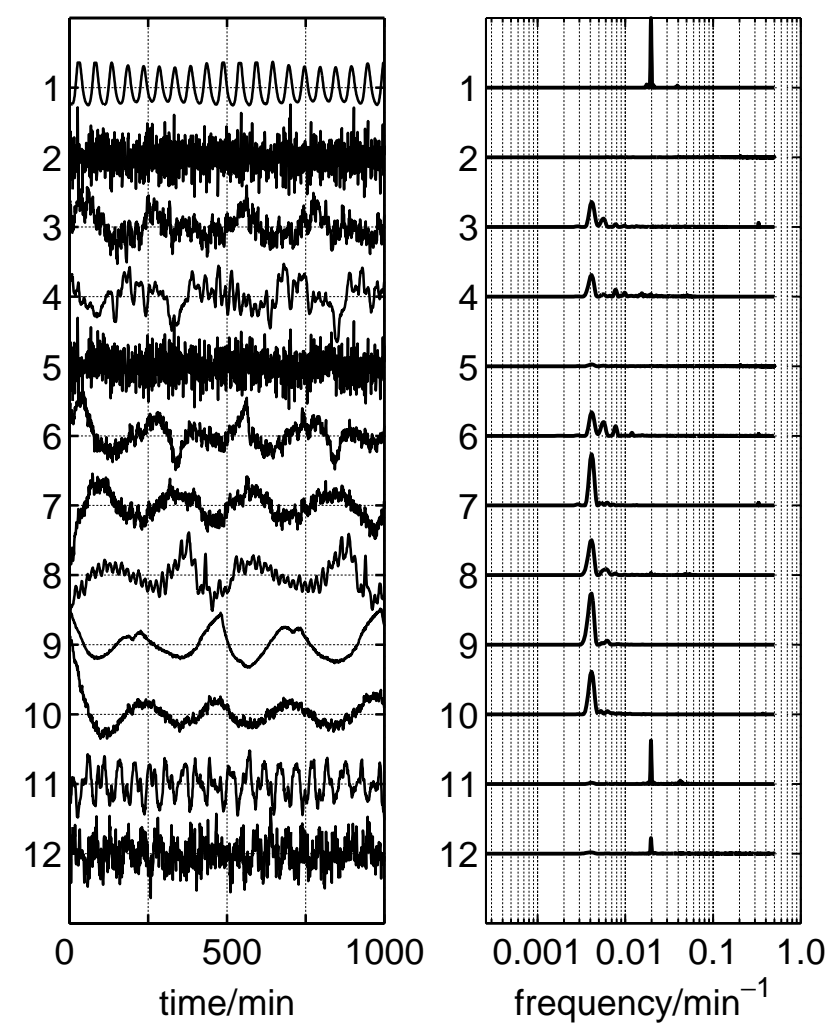

Fig. 6. Filtered time trends and spectra for the challenge problem.

spectra was able to demonstrate that fact by removing the dominating influence of the low-frequency oscillation.

The SPE distribution for the filtered spectra showed tag 6 well separated from the main distribution (bottom plot in column (b) of Fig. 5). Thus, PCA using filtered spectra successfully highlighted tag 6 as abnormal. The reason is that the oscillation in its time trend was the most non-sinusoidal and it therefore had higher harmonics beyond the second that were not captured by the new $\boldsymbol{w}_{1}^{\prime}$-vector.

To summarise, PCA using the full spectra achieved a broad-brush analysis which classified the tags into two dominant categories. That insight motivated and guided a more detailed analysis using filtered spectra which revealed additional, more subtle effects including the detection of a tag with a known abnormality.

\subsubsection{PCA using autocovariance functions}

The plots in column (c) of Fig. 5 show the results from PCA using autocovariance functions. The weightings and $S P E$ plots were very similar to those from spectral PCA (column (a)) with the tags arranged along the two orthogonal axes. The $\boldsymbol{w}^{\prime}$-vectors resembled autocovariance functions having oscillations with a period of 500 and $50 \mathrm{~min}$. Note that the oscillations in the $\boldsymbol{w}^{\prime}$-vectors have zero phase lag because the calculation of the 
autocovariance removed the phase information that was present in the original time trends. Tags 3-10 and 12 lay on the horizontal axis because their autocovariance functions resemble the $\boldsymbol{w}^{\prime}{ }_{1}$-vector while tags 1 and 11 were close to the vertical axis of the score plot because their autocovariance functions resemble the $\boldsymbol{w}_{2}{ }_{2}$-vector. The noisy tag 2 was plotted to the origin because its flat autocovariance function (see Fig. 2) was not modelled by either $\boldsymbol{w}_{2}^{\prime}$-vector and tag 5 was also near the origin because it was more noisy than other tags.

Table 2 shows that the amount of variance in the data set explained by the $\boldsymbol{w}_{1}{ }_{1}$ - and $\boldsymbol{w}_{2}{ }_{2}$-vectors in PCA using autocovariance functions was identical to that achieved with spectral PCA using the same number of channels in the spectrum as lags in the autocovariance function (967 in this case, half of the total number of data samples). The reason why their performance was the same is that no smoothing or averaging was applied during the Fourier transform calculations. Therefore, the spectra and autocovariance functions encoded the same information because the power spectrum is a linear integral transform of the autocovariance function.

Acoustic emissions were analysed using spectral PCA in another project (Tan, Thornhill, \& Belchamber, 2002) in which each time trend contained 44000 samples. The data set was too large to work with and thus the transformed data matrix was reduced to 1024 channels. A generic result found in that work is of relevance to spectral and autocovariance PCA of process data and is therefore mentioned here. Smoothed spectra were computed in 1024 frequency channels using the averaged periodogram method of Welch (1967). For the autocovariance functions, however, complete two-sided spectra with 22000 frequency channels (positive and negative) were calculated with no averaging and the first 1024 lags of their inverse Fourier transforms were used for autocovariance PCA. In that case, PCA using the autocovariance functions required only two $\boldsymbol{w}^{\prime}$-vectors, while the model for spectral PCA needed three to capture the same amount of variation. It was concluded that if there is a need to reduce the size of the data matrix (the $\boldsymbol{X}$ matrix) then autocovariance PCA performs better than PCA using smoothed spectra.

\subsection{Results from the refinery example}

\subsubsection{Major clusters in spectral PCA}

Table 2 gives the percentage variance explained by spectral PCA in the refinery data set. Three principal components described $90 \%$ of the variance. Fig. 7 shows the PCA scores plot for the spectra of the 37 tags from the refinery example. The left-hand plot shows all the tags and the right-hand plot shows a magnified view of the central cluster. They are three-dimensional plots because the model needed three $\boldsymbol{w}^{\prime}$-vectors. Fig. 7 also shows the $\boldsymbol{w}_{1}^{\prime}{ }^{-}, \boldsymbol{w}_{2-}^{\prime}$ and $\boldsymbol{w}_{3}^{\prime}{ }_{3}$-vectors and the $S P E$ histogram.

The white squares in Fig. 7 represent a family of tags with positive $t_{1}$ scores. Therefore their spectra were dominated by the $\boldsymbol{w}^{\prime}{ }_{1}$-vector and had a spectral peak with a frequency of $0.06 \mathrm{~min}^{-1}$ (an oscillation period of $16.7 \mathrm{~min})$. These were tags $2-4,10,11,13,19,20,24,25$, 33 and 34. Observation of the time trends of the tags would classify tags $2-4,10,11,20,34$ and perhaps 33,19 and 13 as having the $0.06 \mathrm{~min}^{-1}$ oscillation. It is unlikely that the time trends of 24 and 25 would be included by visual inspection of the time trends because they are noisy, but the score plot shows that they have positive $t_{1}$
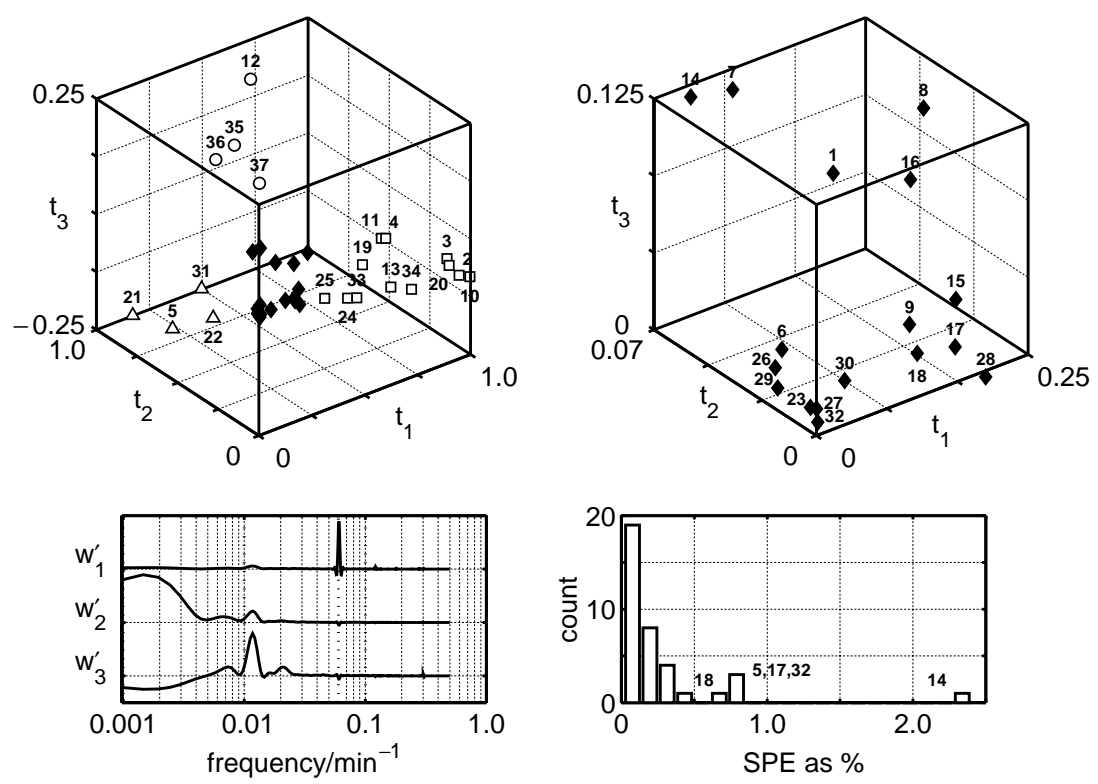

Fig. 7. Score plots (top), $\boldsymbol{w}^{\prime}$-vectors (lower left) and $S P E$ histograms (lower right) for the refinery example. 
scores and thus share the $0.06 \mathrm{~min}^{-1}$ oscillation. The conclusion is that spectral PCA showed there were additional members of the group. These oscillatory tags were associated with the off-gas recycle of the reformer unit (see Fig. 3) and the disturbance was attributed to a faulty control loop within that recycle. The tail gas flow (tag 25) also showed the same disturbance. The propagation to tag 25 may be caused by another recycle from a point downstream of the plant.

A second distinct cluster of tags is the white triangles shown by the spectral PCA plot to have positive values of $t_{2}$ and negative values of $t_{3}$. These were tags $5,21,22$ and 31 characterised by a weighted difference between the $\boldsymbol{w}_{2}^{\prime}$ - and $\boldsymbol{w}_{3}^{\prime}$-vectors. Therefore, the spectra of those tags had a significant component at very low frequency. An inspection of Fig. 4 suggests that it is unlikely that one would group these tags together by visual observation of the time trends of their controller errors. However, Fig. 7 shows their spectra all share a lowfrequency feature which means they all had long-term drifts and deviations from zero. The conclusion is that spectral PCA highlighted an effect that was not obvious by eye. It identified tags belonging to controllers that were not regulating the loop to the set point.

Tag 5 in the above group has a larger $S P E$ value than the others meaning that it had some spectral features that were different from the others in the group. The explanation can be seen in the magnified plots of Fig. 8 which shows that tags 21,22 and 31 had little spectral content above $0.004 \mathrm{~min}^{-1}$. The large low-frequency spectral feature of tag 5, however, extended up to $0.007 \mathrm{~min}^{-1}$, a difference that is reflected in the SPE.

The cluster of circle symbols containing tags 12 and 35-37 had positive values of $t_{2}$ and $t_{3}$ and were thus

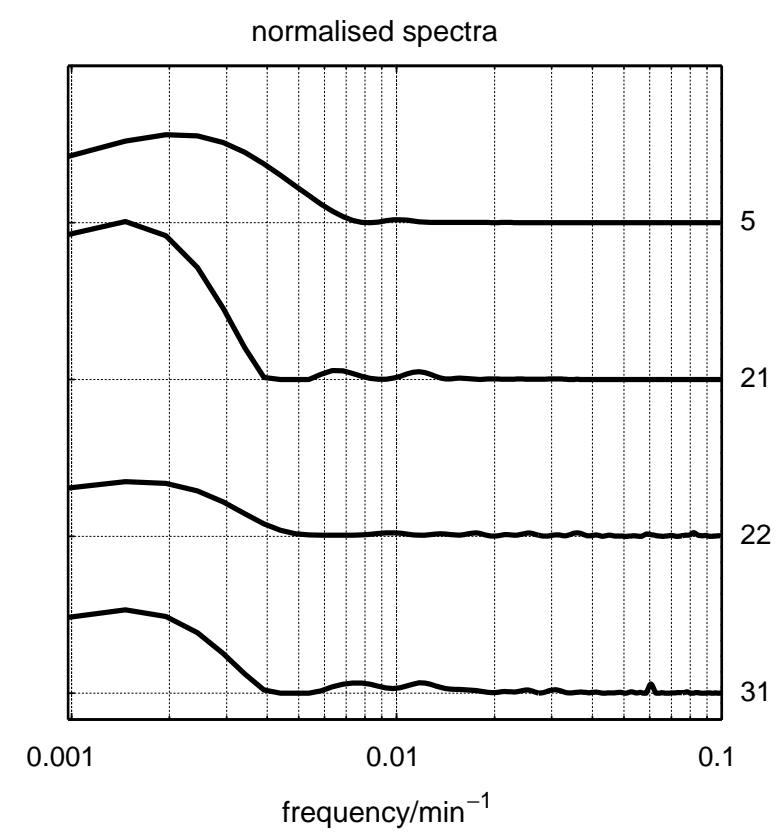

Fig. 8. Magnified spectra for the refinery example. characterised by a weighted sum of the $\boldsymbol{w}_{2}^{\prime}$ - and $\boldsymbol{w}_{3}^{\prime}$ vectors. Those tags therefore had a series of spectral peaks at around $0.01 \mathrm{~min}^{-1}$ and were not pure sinusoids because they contained several spectral components. A visual classification of the time trends would highlight the same group because their oscillations look quite similar to one another. The conclusion is that in this case spectral PCA confirmed a visual classification and showed that there were no other tags having that behaviour. The schematic shows that these loops related to the thermodynamic properties of the fuel gas added to the feed vaporiser. Thus the $0.01 \mathrm{~min}^{-1}$ disturbance can be attributed to the upstream supply of fuel gas.

\subsubsection{Minor clusters in spectral PCA}

The magnified scores plot in Fig. 7 suggests that tags $9,15,17,18$, and 28 form a cluster, but that impression is misleading because tags 17 and 18 have relatively large $S P E$ values and therefore are projections into the threedimensional space from points residing in a higher PCA dimension. Therefore the proposed cluster would actually be only tags 9,15 and 28 .

The cluster near the coordinates $(0,0,0)$ comprises tags $6,23,26,27,29,30$. (tag 32 had a large $S P E$ value and therefore was not a member of the group). Their $t_{1}, t_{2}$ and $t_{3}$ scores were all close to zero because the magnitudes of their normalised spectra were small, and the reason for this is that they have spectral features spread uniformly across a wide range of frequencies. The uniform broad-band spectrum indicates that these tags comprised mainly random noise. This conclusion reinforces the observation from the challenge example, which was that tags characterised by random noise map to the origin of a spectral PCA scores plot. Tags 23, 26, 27, 29 and 30 are critical flow loops while 6 is a pressure control loop in the process gas line. These control loops would be tuned for accuracy and should have a random error signal with no predictable features such as oscillations or steady offsets (Harris, 1989). Therefore the conclusion can be restated: well-controlled loops map to the origin of the spectral PCA score plot.

The remaining cluster comprised tags $1,7,8$ and 16 (14 had a large $S P E$ and was not a member). They were quite close to the origin because their time trends were noisy, but the scores plot in Fig. 7 shows they had positive values of $t_{1}$ and $t_{3}$ and thus featured the spectral peaks at around 0.01 and $0.06 \mathrm{~min}^{-1}$ oscillation. Therefore spectral PCA analysis shows they were disturbed by the plant wide oscillation in the off gas recycle and also by the disturbance in the thermodynamic properties of the fuel gas.

Tags 5, 14, 17, 18 and 32 had the largest $S P E$ values. Tag 5 has already been discussed. In the case of tags 14 , 17,18 and 32 the $S P E$ reflected the fact that they had frequency components at frequencies that were not captured by the three $\boldsymbol{w}^{\prime}$-vectors of the model. For 
instance, tag 32 had an oscillation at a frequency of just under $0.2 \mathrm{~min}^{-1}$. It required a model with seven principal components to model that feature, which was captured by the $\boldsymbol{w}_{7}^{\prime}$-vector.

\section{Comparison of time shift and spectral PCA}

Table 2 shows that the use of PCA directly on the time trends (time domain PCA) in the refinery data set performed poorly. The first three terms in the model described only $41 \%$ of the variability in the data set. Fig. 9 shows the scores plot of the time domain PCA analysis and the $\boldsymbol{w}^{\prime}$-vectors, which are time trends. Clusters such as the white squares that were clear in spectral PCA analysis did not emerge in the time domain PCA analysis. The white squares representing tags with the $0.06 \mathrm{~min}^{-1}$ were all close to the $t_{2}=0$ plane in Fig. 9 and had large magnitudes of $t_{1}$ or of $t_{3}$, or of both. They were therefore characterised by various weighted combinations of the $\boldsymbol{w}_{1}{ }_{1}$ - and $\boldsymbol{w}_{3}{ }_{3}$-vectors. These are oscillatory time domain functions with a frequency of $0.06 \mathrm{~min}^{-1}$ (oscillation period of $16.7 \mathrm{~min}$ ) and are orthogonal. The explanation for the lack of clustering of the square symbols is that both the $\boldsymbol{w}_{1}{ }_{1}$ - and $\boldsymbol{w}_{3}{ }_{3}$ vectors were needed in various amounts to reconstruct $0.06 \mathrm{~min}^{-1}$ oscillations of arbitrary phase. In spectral PCA, by contrast, the square symbols were clustered together because they all had a spectral peak at $0.06 \mathrm{~min}^{-1}$. These results prove that spectral PCA gives better performance than time domain PCA when process dynamics and time delays cause phase shifts in the time trends, and indicate why time domain PCA requires more principal components for the same data set

A further study was conducted using time shifted PCA on a subset of the refinery data. The tags used were $2-4,10,20$ and 34 . The reason for selecting these tags was that they showed the clearest $0.06 \mathrm{~min}^{-1}$ oscillation and their time shifts could be easily determined. The lefthand plot of Fig. 10 shows the selected time trends where it can be seen that the oscillations were not in phase and did not align. It also marks with a dot the time delays determined by the arguments of the Fourier transforms at the frequency of oscillation, as described in the Methods section. For instance, the arguments of the Fourier transform of tags 2 and 3 at $0.06 \mathrm{~min}^{-1}$ were 0.165 and $-1.043 \mathrm{rad}$, respectively. Therefore the phase of tag 3 relative to tag 2 was $-1.208 \mathrm{rad}$ and the time delay of tag 3 relative to tag 2 was

$T_{d}=\frac{1.208}{2 \pi \times 0.06}=3.204 \mathrm{~min}$.
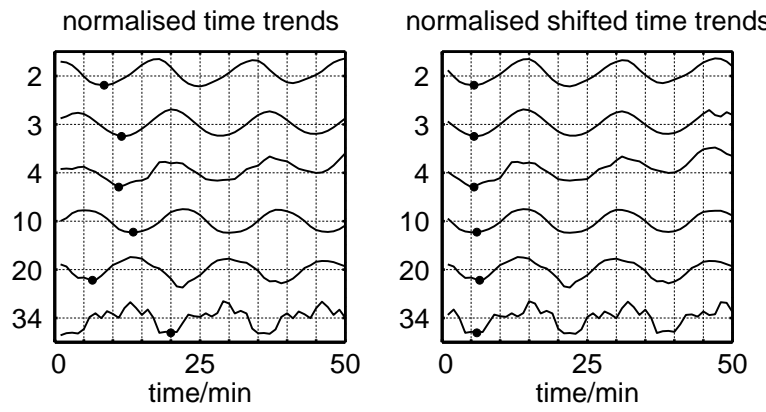

Fig. 10. Alignment of selected trends from the refinery problem for time shifted PCA.
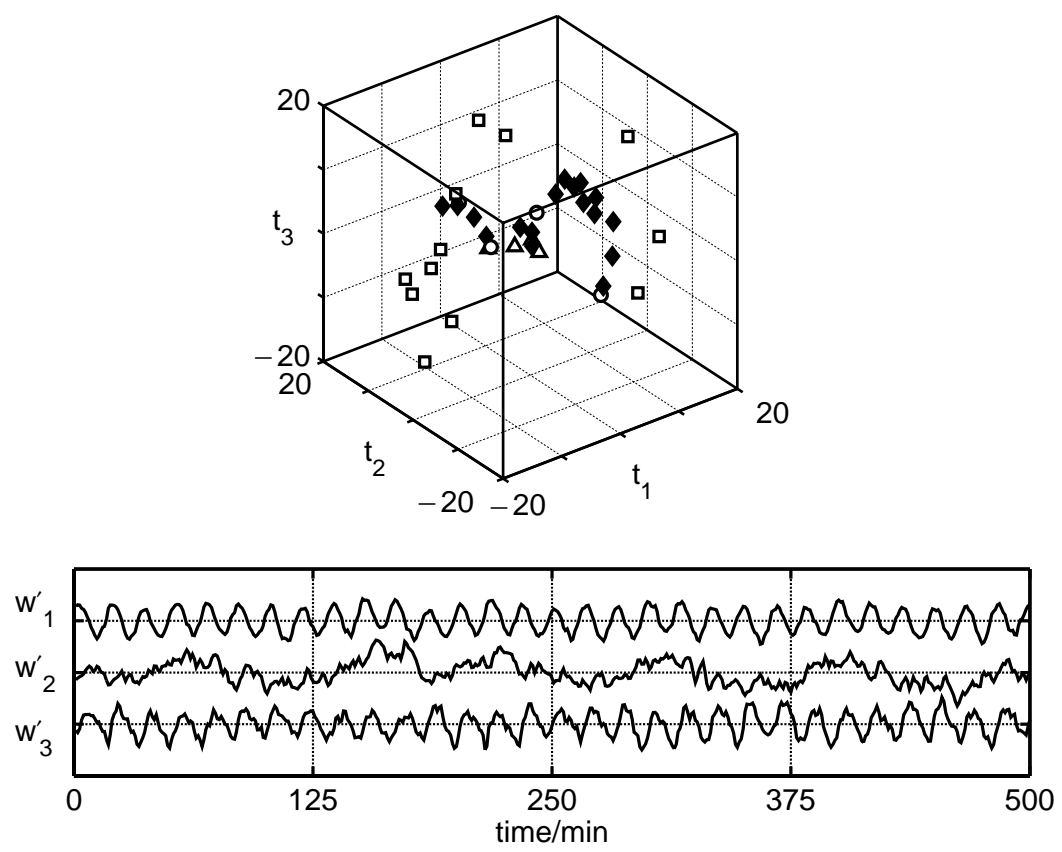

Fig. 9. Time domain PCA for the refinery example. 
The right-hand plot shows the time trends after time shifting in which it can be seen that the delays determined from the Fourier transforms aligned the peaks and valleys.

A comparison was made of time domain PCA without time shifted vectors, PCA with time shifted vectors, and spectral PCA for these six tags. The models required three, two and one principal components, respectively (Table 2). Therefore spectral PCA outperformed time shifted PCA because it captured the variability in the data set with fewer principal components and both were better than time domain PCA with no time shifting.

It might be expected, as with autocovariance PCA, that the time shifted PCA should have identical performance to spectral PCA because all these methods remove phase information. Therefore the finding that spectral PCA had superior performance needs an explanation. The reason is that the phase information was not fully removed by time shifting because time shifts can only be applied in integer multiples of the sampling interval. Hence, for instance, the time delay determined for tag 3 relative to tag 2 was $3.20 \mathrm{~min}$, but the time shift applied was only $3 \mathrm{~min}$ being the closest integer number of sampling intervals. It is also noted that, in general, time shifting cannot remove all delays. For instance, suppose that oscillations at two unrelated frequencies originate in control loops $\mathrm{A}$ and $\mathrm{B}$ and propagate plant-wide. Propagation delays from A are not the same as from B because the routes through the plant are different and therefore it is not normally possible to find a unique time shift that aligns both oscillations simultaneously.

\section{Conclusions}

This paper has demonstrated off-line principal component analysis (PCA) using power spectra for cases when the measurements in the data matrix had phase lags or time delays caused by process dynamics. The power spectra were invariant to lags or delays and thus could classify measurements having similar but timedelayed features such as oscillations with phase lags or long-term deviations from the average value.

The performance of spectral PCA was demonstrated in two case studies, one a challenge problem using simulated data of a pulp process and the other a refinery example. Spectral PCA proved able to highlight clusters of tags showing common features and discovered other tags that were members of the clusters that could not be identified by visual inspection. An advantage of spectral PCA was the easy removal of unwanted interferences to highlight subtle effects. Thus, removal of the spectral peak of a large amplitude plant-wide oscillation enabled more detailed analysis of features with smaller amplitudes.
PCA using autocovariance functions was also studied. When no smoothing of the spectra was used then the performances of autocovariance PCA and spectral PCA were identical and the same number of principal components captured the same amount of variability in the data matrix. It is known, however, that if an averaged periodogram in fewer frequency channels were used then PCA using autocovariance functions would require fewer principal components for the same size of data matrix and therefore has superior performance.

Spectral PCA had superior performance to time domain PCA for the detection of clusters of data trends having similar features, even when time shifting was used to align the data trends. The reason why time shifting did not fully solve the problem is that sampled data vectors can only shift by an integral number of sampling intervals, whereas the time delays or phase lags to be removed may not be an integral number of sampling intervals.

\section{Acknowledgements}

Nina Thornhill gratefully acknowledges the financial support of the Royal Academy of Engineering (Foresight Award). The authors are grateful for the support of the Natural Science and Engineering Research Council (Canada), Matrikon (Edmonton, Alberta) and the Alberta Science and Research Authority through the NSERC-Matrikon-ASRA Industrial Research Chair in Process Control. The challenge problem was provided courtesy of EnTech Control Inc. (Toronto, Ont., Canada).

\section{References}

Åström, K. J. (1991). Assessment of achievable performance of simple feedback loops. International Journal of Adaptive Control and Signal Processing, 5, 3-19.

Bakshi, B. R. (1998). Multiscale PCA with application to multivariate statistical process monitoring. A.I.Ch.E. Journal, 44, 1596-1610.

Bakshi, B. R., \& Stephanopoulos, G. (1994). Representation of process trends-IV. Induction of real-time patterns from operating data for diagnosis and supervisory control. Computers in Chemical Engineering, 18, 302-332.

Belchamber, R. M., \& Collins, M. P. (1993). Method for monitoring acoustic emissions. European Patent Office, Publication No. 0317 $322 \mathrm{~B} 1$.

Bissessur, Y., Martin, E. B., \& Morris, A. J. (1999). Monitoring the performance of the paper making process. Control Engineering Practice, 7, 1357-1368.

Chatfield, C., \& Collins, A. J. (1980). Introduction to multivariate analysis. London, UK: Chapman \& Hall.

Chen, G., McAvoy, T. J., \& Piovoso, M. J. (1998). A multivariate statistical controller for on-line quality improvement. Journal of Process Control, 8, 139-149.

Cook, P. A. (1986). Nonlinear dynamical systems. London: PrenticeHall International. 
Gollmer, K., \& Posten, C. (1996). Supervision of bioprocesses using a dynamic time warping algorithm. Control Engineering Practice, 4, 1287-1295.

Goulding, P. R., Lennox, B., Sandoz, D. J., Smith, K. J., \& Marjanovic, O. (2000). Fault detection in continuous processes using multivariate statistical methods. International Journal of Systems Science, 31, 1459-1471.

Hägglund, T. (1995). A control-loop performance monitor. Control Engineering Practice, 3, 1543-1551.

Harris, T. (1989). Assessment of control loop performance. Canadian Journal of Chemical Engineering, 67, 856-861.

Harris, T. J., Seppala, C. T., Jofreit, P. J., \& Surgenor, B. W. (1996). Plant-wide feedback control performance assessment using an expert system framework. Control Engineering Practice, 9, 1297-1303.

Jackson, J. E., \& Mudholkar, G. S. (1979). Control procedures for residuals associated with principal components analysis. Technometrics, 21, 341-349.

Karstang, T. V., \& Henriksen, A. (1992). Infrared spectroscopy and multivariate calibration used in quantitative analysis of additives in high density polyethylene. Chemometrics and Intelligent Laboratory Systems, 14, 331-339.

Kassidas, A., Taylor, P. A., \& MacGregor, J. F. (1998). Off-line diagnosis of deterministic faults in continuous dynamic multivariable processes using speech recognition methods. Journal of Process Control, 8, 381-393.

Kosanovich, K. A., \& Piovoso, M. J. (1997). PCA of wavelet transformed process data for monitoring, Intelligent Data Analysis Journal, 1(2). 1, 85-99.

Kourti, T., \& MacGregor, J. F. (1996). Control of multivariate processes. Journal of Quality Control, 28, 409-428.

Kresta, J. V., MacGregor, J. F., \& Marlin, T. E. (1991). Multivariate statistical monitoring of process operating performance. Canadian Journal of Chemical Engineering, 69, 35-47.

Ku, W. F., Storer, R. H., \& Georgakis, C. (1995). Disturbance detection and isolation by dynamic principal component analysis. Chemometrics and Intelligent Lab Syst, 30, 179-196.

Lakshminarayanan, S., Shah, S. L., \& Nandakumar, K. (1997). Modelling and control of multivariable processes: Dynamic PLS approach. A.I.Ch.E. Journal, 43, 2307-2322.

Luo, R. F., Misra, M., \& Himmelblau, D. M. (1999). Sensor fault detection via multiscale analysis and dynamic PCA. Industrial and Engineering Chemistry Research, 38, 1489-1495.

Martin, G. D., Turpin, L. E., \& Cline, R. P. (1991). Estimating control function benefits. Hydrocarbon Processing, 70(6), 68-73.

Negiz, A., \& Cinar, A. (1997). Statistical monitoring of multivariable dynamic processes with state-space models. A.I.Ch.E. Journal, 43, 2002-2020.

Negiz, A., \& Cinar, A. (1998). Monitoring of multivariable dynamic processes and sensor auditing. Journal of Process Control, 8, 375-380.

Press, W. H., Flannery, B. P., Teukolsky, S. A., \& Vetterling, W. T. (1986). Numerical recipes. Cambridge: Cambridge University Press.

Rengaswamy, R., \& Venkatasubramanian, V. (1995). A syntactic pattern-recognition approach for process monitoring and faultdiagnosis. Engineering and Applied Artificial Intelligence, 8, 35-51.

Riley, M. R., Rhiel, M., Zhou, X., Arnold, M. A., \& Murhammer, D. W. (1997). Simultaneous measurement of glucose and glutamine in insect cell culture media by near infrared spectroscopy. Biotechnology and Bioengineering, 55, 11-15.

Seasholtz, M. B. (1999). Making money with chemometrics. Chemometrics and Intelligent Laboratory Systems, 45, 55-64.

Shao, R., Jia, F., Martin, E. B., \& Morris, A. J. (1999). Wavelets and non-linear principal components analysis for process monitoring. Control Engineering Practice, 7, 865-879.

Shi, R. J., \& MacGregor, J. F. (2000). Modeling of dynamic systems using latent variable and subspace methods. Journal of Chemometrics, 14, 423-439.

Stork, C. L., Veltkamp, D. J., \& Kowalski, B. R. (1998). Detecting and identifying spectral anomalies using wavelet processing. Applied Spectroscopy, 52, 1348-1352.

Tabe, H. T., Chow, K. C., Tan, K.-J., Zhang, J., \& Thornhill, N. F. (1998). Dynamic principal component analysis using integral transforms. A.I.Ch.E. Annual Meeting, Miami Beach.

Tan, C. C., Thornhill, N. F., \& Belchamber, R. M. (2002). Principal component analysis of spectra with application to acoustic emissions from mechanical equipment, Transactions of the Institute of Measurement and Control, in press.

Terrible, J., Shahani, G., Gagliardi, C., Baade, W., Bredehoft, R., \& Ralston, M. (1999). Consider using hydrogen plants to cogenerate power needs. Hydrocarbon Processing, 78, 43-53.

Thornhill, N. F., \& Hägglund, T. (1997). Detection and diagnosis of oscillation in control loops. Control Engineering Practice, 5, 1343-1354.

Thornhill, N. F., Huang, B., \& Zhang, H. (2002). Detection of multiple oscillations in control loops, Journal of Process Control, in press.

Valle, S., Li, W. H., \& Qin, S. J. (1999). Selection of the number of principal components: The variance of the reconstruction error criterion with a comparison to other methods. Industrial and Engineering Chemistry Research, 38, 4389-4401.

Wachs, A., \& Lewin, D. R. (1999). Improved PCA methods for process disturbance and failure identification. A.I.ChE. Journal, 45, 1688-1700.

Welch, P. D. (1967). The use of fast Fourier transforms for the estimation of power spectra. IEEE Transactions on Audio and Electroacoustics, AU-15, 70-73.

Wise, B. M., \& Gallagher, N. B. (1996). The process chemometrics approach to process monitoring and fault detection. Journal of Process Control, 6, 329-348.

Wise, B. M., \& Ricker, N. L. (1992). Identification of finite impulse response models by principal components regression: Frequency response properties. Process Control and Quality, 4, 77-86.

Wise, B. M., Ricker, N. L., Veltkamp, D. F., \& Kowalski, B. R. (1990). A theoretical basis for the use of principal components models for monitoring multivariate processes. Process Control and Quality, 1, 41-51.

Wold, S. (1976). Pattern recognition by means of disjoint principal component models. Pattern Recognition, 8, 127-139.

Wold, S., Esbensen, K., \& Geladi, P. (1987). Principal component analysis. Chemometrics and Intelligent Laboratory Systems, 2, 37-52.

Yeung, K. S. Y., Hoare, M., Thornhill, N. F., Williams, T., \& Vaghjiani, J. D. (1999). Near infra red spectroscopy for bioprocess monitoring and control. Biotechnology and Bioengineering, 63, 684-693. 